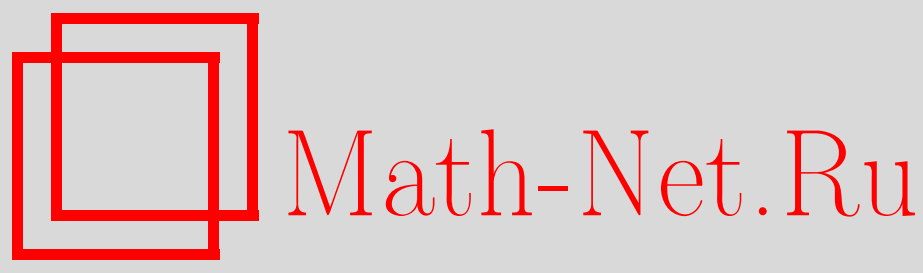

А. Е. Шабад, Сингулярный центр как негравитационная черная дыра, ТМФ, 2014, том 181, номер 3, 603-613

DOI: https://doi.org/10.4213/tmf8749

Использование Общероссийского математического портала Math-Net.Ru подразумевает, что вы прочитали и согласны с пользовательским соглашением http: //www.mathnet.ru/rus/agreement

Параметры загрузки:

IP: 18.209 .158 .208

26 апреля 2023 г., 18:06:35

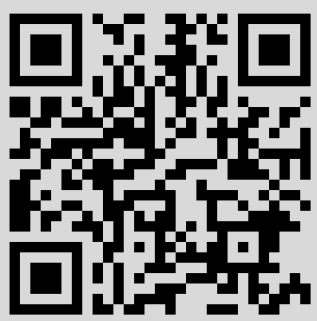




\title{
ФИЗИКА
}

Том 181, № 3

декабрь, 2014

(C) 2014 г.

\author{
А. Е. Шабад*
}

\section{СИНГУЛЯРНЫЙ ЦЕНТР КАК НЕГРАВИТАЦИОННАЯ ЧЕРНАЯ ДЫРА}

\begin{abstract}
Трактовка сингулярного центра в квантовой механике как черной дыры рассматривается в приложении к точно решаемой задаче о трехмерном осцилляторе с комплексным угловым моментом, известной также как обобщенная задача Калоджеро.
\end{abstract}

Ключевые слова: сингулярный потенциал, падение на центр, квантово-механическая черная дыра.

DOI: $10.4213 / \operatorname{tmf} 8749$

\section{1. ПОСВЯЩЕНИЕ}

Давным-давно, когда люди еще не знали механизма Хиггса, сделавшего перенормируемой теорию с промежуточным заряженным векторным бозоном [1], они полагали вслед за Гейзенбергом [2], что нарастание степеней расходимостей от приближения к приближению, характерное для неперенормируемости, может быть излечено, если как-то правильно подойти к квантово-механической задаче с сильно сингулярным потенциалом, обладающей тем же недостатком. Наша, оставшаяся, к сожалению, единственной, совместная работа с А. А. Славновым [3] - в биографии обоих авторов одна из первых, если вообще не первая, - была как раз посвящена этому вопросу: рассмотрению метода суммирования расходящихся вкладов в пропагатор в “теории ператизации” Фейнберга и Пайса [4]. Едва ли это был “шаг в правильном направлении". Квантовая теория поля пошла, и при существенном участии того же А. А. Славнова, по другому пути, но сингулярный потенциал продолжает привлекать к себе внимание. И сейчас я снова обращаюсь к этой теме, теперь уже, правда, не в связи с перенормируемостью, хотя, кто знает ...

\section{2. ВВЕДЕНИЕ}

В работах [5], [6] был предложен подход к задачам квантовой механики с сингулярным (в начале координат) потенциалом притяжения, вызывающим падение на центр [7], в которых сингулярный центр $\sim \beta^{2} / r^{2}$ трактуется как (негравитационная) черная дыра. Решения уравнения Шредингера, осциллирующие в противофазе в окрестности центра, рассматриваются как частицы, асимптотически свободные

* Физический институт им. П. Н. Лебедева РАН, Москва, Россия.

E-mail: shabad@lpi.ru 
при приближении к нему, падающие в центр или исходящие из него. Для осмысленности такой трактовки важно, что упомянутые решения нормируются на дельтафункцию, как это положено свободным частицам. Эта нормировка обеспечивается тем, что то же самое дифференциальное уравнение Шредингера представляется в виде специальной задачи на собственные значения для оператора, отличного от гамильтониана. За физические состояния принимается полный набор, связанный с этой задачей. Мера, с которой ортогональны собственные функции в этой задаче, сингулярна, подобно метрике гравитационной черной дыры. Эта сингулярность заимствована от потенциала, и именно она обеспечивает полезную расходимость, необходимую для нормировки волновой функции на дельта-функцию.

В работах [5], [6] был рассмотрен общий случай потенциала, убывающего на больших расстояниях от центра, так что имеется обычный непрерывный спектр. Было указано на состояния упругого рассеяния "внутрь", когда испущенная из центра частица полностью отражается обратно в центр, а также на двухканальные состояния, которым приписывается унитарная матрица рассеяния размера $2 \times 2$. В последнем случае частица частично отражается в центр, а частично (с определенным коэффициентом прохождения) уходит на бесконечность; также и обратно: падая из бесконечности, частично отражается туда же, а частично проходит, чтобы упасть на сингулярность. Рассмотрение велось в общем виде, и все результаты, включая элементы $S$-матрицы, выражались в терминах функций Йоста. В работе [8] полученная конструкция применялась к задаче об уравнении Дирака в поле точечного заряда $Z>137$, обладающей той же математической природой, и изучалось поглощение электронов центром и рождение свободных позитронов.

В рамках классической механики падению на центр соответствует наличие "инфинитного" движения к нему по логарифмической спирали, накручивающейся на центр и обходящей его бесчисленное количество раз за конечное время. По часам наблюдателя, отсчитывающего время по количеству витков, понадобилась бы вечность для достижения центра.

В настоящей работе указанный круг идей реализуется применительно к точно решаемому случаю с притягивающим потенциалом, неограниченно растущим при удалении от центра. Явно описан континуум состояний упругого рассеяния из центра в центр и ранее не отмеченные дискретные ("распадные") состояния с комплексной энергией, соответствующие поглощению на центре (или испусканию им).

В противоположность нашему подходу традиционным при рассмотрении задач с падением на центр является метод самосопряженного расширения гамильтониана. Соответствующий спектр связанных состояний был впервые обнаружен в работе Кэйса [9] и осмыслен в соответствии с теорией фон Неймана в работе Меетца [10]. Метод был введен в обиход в книгах [11]. Наиболее полное и всестороннее исследование применительно к разным задачам квантовой механики дано в книге [12]. В этом методе гамильтониан - исходно неограниченный симметрический оператор - доопределяется так, чтобы стать самосопряженным за счет необходимого расширения его области определения до совпадения с (исходно другой) областью определения сопряженного оператора. Самосопряженное расширение многозначно. Формулировка содержит условия фиксации произвола, аналогичные выбору граничных условий в регулярной задаче. Метод предполагает решение проблемы выбора, исходящей из сторонних, физически мотивированных постулатов. 
Коренное отличие нашего подхода в том, что физические состояния связываются с собственными функциями не гамильтониана, а другого дифференциального оператора в рамках того же дифференциального уравнения Шредингера, приведенного к виду специальной задачи на собственные значения для этого оператора. В итоге образуется непрерывный спектр состояний рассеяния в сингулярный центр и из него в области параметров, где метод самосопряженного расширения дает, напротив, дискретный спектр. Дальнейшие сравнительные замечания применительно к частному случаю, рассмотренному здесь, сделаны в п. 3.1.

\section{3. ЗАДАЧА ОБ ОБОБЩЕННОМ ГАРМОНИЧЕСКОМ ОСЦИЛЛЯТОРЕ}

Расмотрим одномерное уравнение Шредингера на полупрямой $z>0$

$$
-\frac{d^{2} \eta(z)}{d z^{2}}+\left[z^{2}+\frac{1}{z^{2}}\left(\gamma-\frac{1}{2}\right)\left(\gamma-\frac{3}{2}\right)\right] \eta(z)=(4 \alpha+2 \gamma) \eta(z),
$$

которое можно получить [7] как радиальную часть трехмерного уравнения Шредингера со сферически-симметричным притягивающим потенциалом осциллятора $U(r)=\left(m \omega^{2} / 2\right) r^{2}$, где $m$ - масса, $\omega$ - частота и $r$ - длина радиус-вектора, при нецелых вещественных и комплексных значениях углового момента $l=\gamma-3 / 2$. Такое же уравнение известно как одномерная задача Калоджеро [13].

Дифференциальный оператор (точнее, дифференциальную операцию) в левой части уравнения (1) будем называть "гамильтонианом“. Решение уравнения (1) связано с решением уравнения для вырожденной гипергеометрической функции

$$
x y^{\prime \prime}+(\gamma-x) y^{\prime}+\alpha y=0
$$

(здесь $x=z^{2}$, штрих означает диференцирование по $x$ ) следующим образом:

$$
\eta(z)=\Phi(z) y\left(z^{2}\right), \quad \Phi(z)=z^{\gamma-1 / 2} e^{-z^{2} / 2}
$$

Уравнение (1) является нормальной формой Лиувилля для уравнения (2). Собственное значение энергии $E$ связано с параметрами уравнения (1):

$$
\frac{E}{\hbar \omega}=2 \alpha+\gamma
$$

где $\omega$ - частота.

Одно решение вырожденного гипергеометрического уравнения (2), нормированное, как функция Куммера,

$$
y_{1}(x)=M(-\alpha, \gamma ; x)=1-\frac{\alpha}{\gamma} x+\frac{\alpha(\alpha-1)}{\gamma(\gamma+1)} \frac{x^{2}}{2}-\frac{\alpha(\alpha-1)(\alpha-2)}{\gamma(\gamma+1)(\gamma+2)} \frac{x^{3}}{3 !}+\cdots
$$

недопустимо растет при $x \rightarrow \infty$ [14]:

$$
y_{1}(x) \sim \frac{\Gamma(\gamma)}{\Gamma(-\alpha)} x^{-(\alpha+\gamma)} e^{x}
$$


Как видно из соотношений (5), (6), этот рост не происходит при целочисленных значениях

$$
\alpha=n, \quad n=0,1,2, \ldots,
$$

при которых функции Куммера превращаются в многочлены, ортогональные с мерой $\left(x^{\gamma-1} / 2\right) e^{-x} d x$.

Второе решение тоже выражается через функцию Куммера:

$$
\begin{aligned}
y_{2}(x) & =x^{1-\gamma} M(1-\alpha-\gamma, 2-\gamma ; x)= \\
& =x^{1-\gamma}\left(1-\frac{\alpha+\gamma-1}{2-\gamma} x+\frac{(\alpha+\gamma-1)(\alpha+\gamma-2)}{(2-\gamma)(3-\gamma)} \frac{x^{2}}{2}+\cdots\right) .
\end{aligned}
$$

Оно также растет пропорционально $e^{x}$. Но ряд в (8) можно оборвать, тогда он превращается в многочлен, при целых значениях параметра $\alpha+\gamma$,

$$
\alpha=n^{\prime}-\gamma, \quad n^{\prime}=1,2, \ldots,
$$

так что решение $y_{2}$ есть многочлен, умноженный на $x^{1-\gamma}$.

В окрестности точки $z=0$ первое решение $\eta_{1}(z)=\Phi y_{1}$ ведет себя как

$$
\eta_{1}(z) \sim z^{\gamma-1 / 2}
$$

а второе, $\eta_{2}(z)=\Phi y_{2},-$ как

$$
\eta_{2}(z) \sim z^{3 / 2-\gamma}
$$

3.1. Центробежное отталкивание и докритическое притяжение. Стандартный подход.

3.1.1. Сильное отталкивание. В областях

$$
-\infty<\gamma<0, \quad 2<\gamma<\infty
$$

в окрестности нуля имеется сильный отталкивающий потенциал, который вместе с притягивающим осцилляторным потенциалом, доминирующим вдали от центра, создает потенциальную яму. Следовательно, должен быть дискретный спектр связанных состояний. Он состоит из одной из серий (7) или (9). Действительно, если $\gamma>2$, утрачивается квадратичная интегрируемость решения (8), поскольку фактор (11) становится сингулярнее, чем $1 / \sqrt{z}$. В то же время фактор (10) остается квадратично-интегрируемым. Тем самым из спектра выпадает бесконечное счетное множество значений (9). Если же $\gamma<0$, то, наоборот, из спектра выпадает серия (7).

3.1.2. Умеренное отталкивание и докритическое притяжение. Иначе обстоят дела в области $0<\gamma<2$, включающей в себя как области отталкивания $1 / 2 \geqslant \gamma>0$, $2>\gamma \geqslant 3 / 2$, так и область притяжения $1 / 2 \leqslant \gamma \leqslant 3 / 2$ в окрестности начала координат (значение $\gamma=1$, допускающее логарифмическое решение, нами не рассматривается). Здесь оба решения квадратично-интегрируемы,

$$
\int_{0}^{\infty} \eta^{2}(z) d z<\infty .
$$

Поэтому два набора (9) и (7) объединяются. 
Однако линейная комбинация (см. формулу 13.1.3 в справочнике [14]) двух решений (5) и (8)

$$
\begin{aligned}
y_{\mathrm{u}}(x) & =U(-\alpha, \gamma ; x)= \\
& =\frac{\pi}{\sin \pi b}\left(\frac{M(-\alpha, \gamma ; x)}{\Gamma(1-\alpha-\gamma) \Gamma(\gamma)}-x^{1-\gamma} \frac{M(1-\alpha-\gamma, 2-\gamma ; x)}{\Gamma(-\alpha) \Gamma(2-\gamma)}\right)
\end{aligned}
$$

является квадратично-интегрируемым решением уравнения (1) при любых $\alpha$, так как согласно формуле 13.5 .2 в [14] при $x \rightarrow \infty$ мы имеем $^{1)}$

$$
U(-\alpha, \gamma ; x)=x^{\alpha}+x^{\alpha-1}(1-\alpha-\gamma) \alpha
$$

(Для проверки последнего утверждения достаточно подставить (14) в уравнение (2) и убедиться, что оно удовлетворяется в асимптотической области в двух лидирующих порядках $x^{\alpha}$ и $x^{\alpha-1}$.) Тем самым квадрат нормы

$$
\int^{\infty} \eta_{\mathrm{u}}^{2}(z) d z=\frac{1}{2} \int^{\infty} y_{\mathrm{u}}^{2}(x)\left(\frac{x}{a}\right)^{\gamma} x^{-1} e^{-x} d x
$$

сходится также и на верхнем пределе ${ }^{2)}$. Таким образом, отсутствует естественное требование, которое могло бы исключить решение (13).

Наложение нулевого граничного условия $\eta(0)=0$, соответствующего введению бесконечной отталкивающей стенки в начале координат, исключает решение (5) (тем самым и решение (13)), а вместе с ним и спектральную серию (7) при $\gamma \leqslant 1 / 2$. Решение (8) и соответствующая спектральная серия (9) исключаются при ${ }^{3)} \gamma \geqslant 3 / 2$ (значение $\gamma=2$ не рассматриваем). Таким образом, самосопряженность гамильтониана и гильбертовость пространства состояний (его собственных функций) достигаются - с учетом уже описанной выше ситуации вне области $0<\gamma<2$ - везде, где сингулярный (“центробежный”) член в уравнении (1) положителен, т. е. всюду в областях отталкивания $-\infty<\gamma \leqslant 1 / 2,3 / 2 \leqslant \gamma<\infty($ при $\gamma \neq 2)$.

Нулевое граничное условие обычно налагается исходя из требования, чтобы волновая функция $\psi(z)=\eta(z) / z$ трехмерного уравнения Шредингера, из которого могло произойти уравнение (1), была конечна в начале координат [7]. Однако, если исходить из самого уравнения (1), это требование является посторонним, доопределяющим. Но даже нулевое граничное условие не в состоянии исключить решение (13) в области докритического притяжения $1 / 2 \leqslant \gamma \leqslant 3 / 2($ при $\gamma \neq 1$ ). Для этого потребовалось бы более сильное условие, что $\eta(z)$ убывает быстрее, чем $\sqrt{z}$ при малых $x$ :

$$
\eta(z)<z^{1 / 2}
$$

\footnotetext{
1) Поскольку в окрестности $x=\infty$ член в уравнении (1), пропорциональный $1 / z^{2}$, меньше всех остальных, первый (ведущий) член в асимптотическом разложении (14) остается тем же в задачах без сингулярного члена, $\gamma=1 / 2,3 / 2$, когда вместо (13) возникает функция параболического цилиндра.

2) В подобласти $\gamma>2$ рассмотренной выше области (12) второй член в (13) недопустимо ведет себя в окрестности точки $x=0$. Он устраняется наложением требования $\Gamma(-\alpha)=\infty$, и мы снова получаем дискретную серию связанных состояний (7). Аналогично, в подобласти $\gamma<0$ воспроизводится серия (9).

3) Для "физических"' значений $\gamma=l+3 / 2$, где $l=0,1,2, \ldots$, остающаяся серия (7) дает согласно (4) все полуцелочисленные значения $E / \hbar \omega \geqslant 3 / 2$. Низшая энергия равна $3 \hbar \omega / 2$ в соответствии с тем, что исходный осциллятор имеет три степени свободы.
} 
Тогда для выживающего в области $1 / 2 \leqslant \gamma<1$ решения функция $\psi_{2}(x)=\eta_{2}(z) / z$ менее сингулярна, чем $x^{-1 / 2}$; для решения, выживающего в области $1<\gamma \leqslant 3 / 2$, функция $\psi_{1}(x)=\eta_{1}(z) / z$ тоже менее сингулярна, чем $x^{-1 / 2}$. Физическая обоснованность таких требований не ясна, но во всяком случае они обеспечивают самосопряженность гамильтониана и дискретный спектр. В следующем пункте, где будет рассматриваться область закритического притяжения, естественным образом возникнет сингулярная мера, и требование квадратичной интегрируемости решений с этой мерой, приложенное к рассматриваемой сейчас области, эквивалентно условию (15).

Самосопряженное расширение гамильтониана, эквивалентное по результату, по крайней мере на уровне регуляризованной задачи, дополнению потенциала в нуле локальным дельтаобразным взаимодействием, достраивающим необходимую потенциальную яму [15], приводит к утверждению [12], [16], что линейная комбинация решений

$$
\eta_{1}(z) \cos \nu+\eta_{2}(z) \sin \nu
$$

с числом $\nu$ из промежутка $-\pi / 2<\nu<\pi / 2$, параметризующим неоднозначность самосопряженного расширения, образует полный набор собственных функций дискретного спектра со значениями энергии $E_{n}(\nu)$, зависящими от этого параметра. При значениях $\nu=\pi / 2$ и $\nu=0$ воспроизводятся спектральные серии (7) или (9) соответственно (при всяком $\nu$ имеется только одна серия, но не (7) и не (9) вместе). Тем самым эти частные значения самосопряженного расширения соответствуют выбору граничных условий вида (15), обсуждавшихся выше.

3.2. Специальный подход к падению на центр. В области $\operatorname{Re} \gamma=1, \operatorname{Im} \gamma=$ $\beta \gtrless 0$ (значение $\beta=0$ не рассматривается) закритического притяжения, характеризующегося падением на центр, мы применим специальный подход для выделения физических решений, связанный с представлением о сингулярном центре как о черной дыре.

Запишем уравнение (1) с учетом (4) в виде задачи на собственные значения для параметра $\beta$,

$$
-\frac{d^{2} \eta}{d z^{2}}+\left(-\frac{2 E}{\hbar \omega}+z^{2}-\frac{1}{4 z^{2}}\right) \eta=\frac{\beta^{2}}{z^{2}} \eta
$$

в которой мы будем в первую очередь интересоваться вещественными значениями $E$, хотя о комплексных $E$ речь пойдет тоже. Надо подчеркнуть, что оператор, стоящий в левой части этого уравнения, - это не гамильтониан. Мы будем связывать физические состояния с его спектром. Отметим, что задача о собственных значениях относительно параметра, входящего умноженным на некую функцию, является вполне традиционной в контексте теории дифференциальных уравнений [17]. В работе [6] показано, что возникающая система собственных функций полна, и выписано фурье-разложение по ней. Что касается квантовой механики, любопытно отметить, что еще в основополагающих работах Шредингера вариант такой задачи на собственные значения обсуждался, хотя впоследствии, до работ [5], [6], [8], насколько автору известно, не использовался. 
Рассмотрим решение $\eta_{\mathrm{u}}=\Phi y_{\mathrm{u}}$, где $y_{\mathrm{u}}$ дается формулой $(13)$, в закритической области. Около начала координат оно есть линейная комбинация двух волн - выходящей из центра и падающей в него:

$$
\begin{aligned}
\eta_{\mathrm{u}} & \sim \frac{\pi}{\sin \pi \gamma}\left(\frac{z^{\gamma-1 / 2}}{\Gamma(1-\alpha-\gamma) \Gamma(\gamma)}-\frac{z^{3 / 2-\gamma}}{\Gamma(-\alpha) \Gamma(2-\gamma)}\right)= \\
& =\frac{i \pi}{\operatorname{sh} \pi \beta}\left(\frac{z^{i \beta} \sqrt{z}}{\Gamma(1 / 2-E / 2 \hbar \omega-i \beta / 2) \Gamma(1+i \beta)}-\frac{z^{-i \beta} \sqrt{z}}{\Gamma(1 / 2-E / 2 \hbar \omega+i \beta / 2) \Gamma(1-i \beta)}\right) .
\end{aligned}
$$

Это выражение вещественно. Поэтому поток вероятности, задаваемый определителем Вронского $\eta \frac{d \eta^{*}}{d z}-\eta^{*} \frac{d \eta}{d z}$ между некоторым решением и другим решением, получающимся из него комплексным сопряжением, равен нулю. Поскольку в уравнении (16) нет первой производной, так определенный детерминант Вронского не зависит от $z$ [17]. Таким образом, поток вероятности, переносимый решением $\eta_{\mathrm{u}}=\Phi y_{\mathrm{u}}$, равен нулю при всех $z$. Заметим, что в согласии с этим фактом асимптотика (14) тоже вещественна. Мы будем вести дело к тому, чтобы получить возможность рассматривать два осциллирующих фактора (17) как частицы, свободные в окрестности сингулярного центра. Тогда решение (13) будет отвечать упругому рассеянию волны, приходящей из точки сингулярности в начале координат, обратно в сингулярность без поглощения вероятности системой. Выберем для определенности один из возможных знаков $\beta$ : пусть $\beta>0$. Имеет смысл представить (17) в виде

$$
\eta_{\mathrm{u}} \sim \frac{i \pi \sqrt{z}}{\operatorname{sh}(\pi \beta) \Gamma(1 / 2-E / 2 \hbar \omega-i \beta / 2) \Gamma(1+i \beta)}\left(z^{i \beta}+S z^{-i \beta}\right),
$$

где число $S$, по модулю равное единице,

$$
S=-\frac{\Gamma(1 / 2-E / 2 \hbar \omega-i \beta / 2) \Gamma(1+i \beta)}{\Gamma(1 / 2-E / 2 \hbar \omega+i \beta / 2) \Gamma(1-i \beta)}, \quad S^{*} S=1,
$$

будет интерпретироваться как унитарная матрица упругого рассеяния на потенциале частицы, приходящей из сингулярности ("черной дыры“) и уходящей обратно туда же.

Будем действовать в полной аналогии с тем, как обычно трактуются свободные волны в непрерывном спектре на бесконечности, когда потенциал там исчезает. Введем ящик со стенкой при $z=z_{\mathrm{box}}$, как угодно приближенной к сингулярности, и наложим нулевое граничное условие на стенке:

$$
\eta_{\mathrm{u}}\left(z_{\mathrm{box}}\right)=0
$$

Тогда из (18) получаем уравнение для квантованных значений параметра $\beta$ в ящике:

$$
2 \beta_{n} \ln z_{\mathrm{box}}=(2 n+1) \pi+\delta\left(\beta_{n}, E\right),
$$

где $\delta(\beta, E)=\arg S$ - фаза упругого рассеяния. При стремлении стенки ящика к сингулярности, при $z_{\mathrm{box}} \rightarrow 0$, дискретные значения параметра $\beta$ сгущаются в континуум. 
Поскольку дифференциальный оператор в левой части уравнения (16) содержит лишь вещественные функции, решения этого уравнения $\eta_{\mathrm{u}}$, соответствующие разным значениям $\beta_{1} \neq \beta_{2}$, взаимно ортогональны с мерой $d z / z^{2}$, как видно из соотношения

$$
\left(\beta_{1}^{2}-\beta_{2}^{2}\right) \int_{z_{\mathrm{box}}}^{\infty} \eta_{\beta_{1}}^{*} \eta_{\beta_{2}} \frac{d z}{z^{2}}=0
$$

Это можно показать, как обычно, интегрированием выражения (16), домноженного слева на комплексно-сопряженную функцию, вычитанием сопряженной конструкции при другом $\beta$ и интегрированием по частям. Возникающие в процессе граничные члены равны нулю,

$$
\left.\left(\eta_{\beta_{2}}^{*} \frac{d}{d z} \eta_{\beta_{1}}-\eta_{\beta_{2}} \frac{d}{d z} \eta_{\beta_{1}}^{*}\right)\right|_{z_{\mathrm{box}}} ^{\infty}=0
$$

в силу убывания функций $\eta$ вместе с их производными на бесконечности в соответствии с формулами (14), (3) и условием (20) на границе ящика. В то же время из (18) видна конечность производных $d \eta / d z$ и $d \eta^{*} / d z$ при $z=z_{\mathrm{box}}$. Исчезновение этих граничных членов при $\beta_{1}=\beta_{2}$ также является достаточным для установления вещественности собственных значений $\beta^{2}$, учтенной при записи формулы $(21)$.

Сингулярность меры $d z / z^{2}$ в начале координат приводит к возможности нормировать осциллирующие в начале координат компоненты решения на дельта-функцию, что и позволяет интерпретировать их как частицы, свободные в "бесконечном объеме" этой точки. Необходимая для формирования дельта-функции бесконечность образуется за счет расходимости интеграла в (21) на нижнем пределе при совпадающих $\beta$. Поэтому для такой нормировки достаточно в этом интеграле учесть только асимптотическое выражение (17). При $z_{\mathrm{box}} \rightarrow 0$

$$
\int_{z_{\mathrm{box}} \rightarrow 0}^{\infty} z^{i\left(\beta_{1}-\beta_{2}\right)} \frac{d z}{z}=\int_{u_{\mathrm{box}} \rightarrow-\infty}^{\infty} e^{i\left(\beta_{1}-\beta_{2}\right) u} d u \rightarrow 2 \pi \delta\left(\beta_{1}-\beta_{2}\right) .
$$

Использованная здесь замена переменной интегрирования $z=e^{u}$ и предела интегрирования $u_{\text {box }}=\ln z_{\text {box }}$ отображает начало координат $z=0$ в $u=-\infty$. В терминах переменной $u$ мы можем говорить о частицах, свободных в бесконечно удаленной области, что эквивалентно использованным выше рассуждениям о свободе в бесконечном объеме, создаваемом сингулярностью в точке $z=0$. Одновременная замена функции

$$
\tilde{\eta}(z)=\frac{1}{\sqrt{z}} \eta(z)
$$

ликвидирует первую производную в дифференциальном уравнении, которая появляется в результате замены переменной, и тем самым восстанавливает его нормальную форму Лиувилля (16),

$$
-\frac{d^{2} \tilde{\eta}(u)}{d u^{2}}+e^{2 u}\left(\frac{2 E}{\hbar \omega}+e^{2 u}\right) \tilde{\eta}(u)=\beta^{2} \tilde{\eta}(u),
$$

а мера после отображения точки сингулярности на минус бесконечность становится плоской,

$$
|\eta(z)|^{2} \frac{d z}{z^{2}}=|\tilde{\eta}(u)|^{2} d u
$$


Такое растяжение сингулярной точки в полуось может служить моделью введения дополнительной (“пятой”) координаты, применяемого в других задачах. Существует, разумеется, много других совместных преобразований координаты и волновой функции, сохраняющих нормальную форму Лиувилля уравнения, но матрица рассеяния (19) ими не затрагивается.

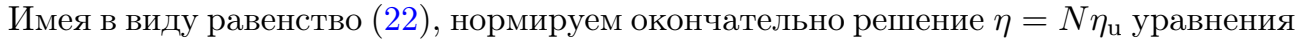
Шредингера (1), (16) так, чтобы каждое из двух слагаемых в (17) было нормировано на дельта-функцию. Тогда нормировочный множитель имеет вид

$$
N=\frac{\operatorname{sh} \pi \beta}{\pi \sqrt{2 \pi}}\left|\Gamma\left(\frac{1}{2}-\frac{E}{2 \hbar \omega}-\frac{i \beta}{2}\right) \Gamma(1+i \beta)\right|,
$$

таким образом,

$$
\eta(z)=\sqrt{\frac{z}{2 \pi}}\left[S^{1 / 2} z^{i \beta} M\left(\Gamma\left(\frac{1}{2}+\frac{E}{2 \hbar \omega}+\frac{i \beta}{2}\right), 1+i \beta ; z^{2}\right)+\text { к. с. }\right] e^{-z^{2} / 2} .
$$

Подчеркнем, что это решение относится ко всем вещественным значениям параметра энергии $E$.

Что касается дискретных серий (7), (9), то соответствующие им значения параметров образуют полюсы и нули матрицы рассеяния (19). Их следует отнести ко второму листу комплексной плоскости переменной $\beta^{2}$. Значения энергии комплексны и попарно комплексно-сопряжены:

$$
\frac{E_{n}}{\hbar \omega}=2 n+1 \pm i \beta, \quad n=0,1,2, \ldots .
$$

При этом волновые функции $\eta_{1}, \eta_{2}$, образованные из многочленов в соответствии c (5), (8), обладают каждая лишь одним осциллирующим поведением в начале координат (вида $z^{i \beta}$ либо $z^{-i \beta}$ ) и поэтому ненулевым потоком вероятности. Это неустойчивые распадные состояния, излучающие в сингулярный центр, и им сопряженные. Такие состояния не были отмечены в предыдущих работах [5], [6], [8], хотя они, разумеется, присутствуют и в рассматривавшихся в указанных работах случаях потенциалов, убывающих при $z \rightarrow \infty$.

Вернемся теперь к областям докритического притяжения и умеренного отталкивания $0<y<2$, в которых требование интегрируемости с квадратом не позволяло выше установить дискретный спектр. Эта цель, однако, достигается, если потребовать квадратичной интегрируемости с естественной в задаче (16) сингулярной мерой $d z / z^{2}$, как в (21). Тогда при $\gamma>1$ выделяется серия дискретного спектра связанных состояний (7), как это уже имело место и с единичной мерой в области сильного отталкивания $\gamma>2$. Аналогично, при $\gamma<1$ наблюдается серия связанных состояний (9).

\section{3. Падение на центр в классике - инфинитное движение к центру.} Квантовым решениям, соответствующим свободным частицам, поглощаемым или испускаемым сингулярным центром, как они были описаны выше, в классике должно соответствовать инфинитное движение в сторону центра. В то время как при вещественном значении углового момента $L$ орбиты в осцилляторном потенциале являются, как известно, замкнутыми, при добавлении к отталкивательному центробежному потенциалу $-L^{2} / m^{2} r^{2}$ сингулярного притягивающего члена $2 \beta^{2} / m r^{2}$, 
существует орбита, спирально накручивающаяся на центр, как только $\beta^{2}>L^{2} / 2 m$. При этом материальная точка совершает вокруг него бесконечное число оборотов за конечное время, проходя конечное расстояние. Действительно, в этом случае начало координат $r=0$ является классически доступной точкой, поскольку величина [18]

$$
R=\left[\left(E-U(r)+\frac{\beta^{2}}{r^{2}}\right) \frac{2}{m}-\frac{L^{2}}{m^{2} r^{2}}\right]^{1 / 2},
$$

где энергия $E>0$, в этой точке вещественна. Из выражения для дифференциала угла

$$
d \phi=R^{-1} \frac{d r}{r^{2}} \frac{L}{m}
$$

следует, что угол при движении по орбите неограниченно логарифмически растет при приближении к сингулярности, $\phi=-(\ln r) L\left(2 \beta m-L^{2}\right)^{-1 / 2}$. С другой стороны, время $t$, необходимое для достижения центра, и расстояние $s$, которое материальная точка проходит прежде, чем она его достигнет, конечны, поскольку дифференциалы $d t=R^{-1} d r$ и $d s=r d \phi$ интегрируемы в точке $r=0$.

Так как осцилляторный потенциал $U(r)=\left(m \omega^{2} / 2\right) r^{2}$ при достаточно больших $r$ доминирует в выражении под корнем в (23), существует предельная точка $r=r_{\max }$ (апогей) такая, что если $r>r_{\max }$, то $R$ становится мнимым. Другими словами, движение является финитным при удалении от центра и инфинитным при приближении к нему. Подобно движению в поле гравитационной черной дыры, от замкнутой орбиты отщепляется спиральная орбита, падающая в дыру.

Стандартное время, исчисляемое в классической механике по количеству замкнутых циклов какого-то процесса, принимаемого в качестве периодического (скажем, движения Земли вокруг Солнца), связано сингулярным преобразованием со временем, в основу отсчета которого положен постулат о “периодичности" движения вокруг сингулярного центра тела, падающего на него по спиральной траектории, т. е. со временем, которое регистрируется наблюдателем, находящимся на падающей траектории.

Благодарности. Автор благодарен И. В. Тютину за обсуждение. Работа поддержана FAPESP в рамках Processo 2014/08970-1 и РФФИ (грант № 14-02-01171).

\section{Список литературы}

[1] G. 't Hooft, Nucl. Phys. B, 35:1 (1971), 167-188.

[2] W. Heisenberg, Z. Physik, 101:9-10 (1936), 533-540; A. T. Filippov, Phys. Lett., 9:1 (1964), 78-80; W. Güttinger, R. Penzl, E. Pfaffelhuber, Ann. Phys. (N. Y.), 33:2 (1965), 246-271.

[3] А. А. Славнов, А.Е. Шабад, ЯФ, 1 (1965), 721-728.

[4] G. Feinberg, A. Pais, Phys. Rev. B, 133:2B (1964), B477-B486.

[5] A. E. Shabad, Singular centre in quantum mechanics as a black hole, arXiv: hep-th/0208133; A. E. Shabad, "Black-hole approach to the singular problem of quantum mechanics", Proceedings of the 3rd International Sakharov Conference on Physics (Moscow, Russia, June 24-29, 2002), 1, eds. A. Semikhatov, M. Vasiliev, V. Zaikin, World Sci., Singapore, 2003, $817-826$.

[6] A. E. Shabad, Black-hole approach to the singular problem of quantum mechanics. II, arXiv: hep-th/0403177.

[7] Л. Д. Ландау, Е. М. Лифшиц, Квантовая механика, Наука, М., 1989. 
[8] A.E. Shabad, J. Phys. A: Math. Gen., 38:33 (2005), 7419-7439, arXiv: hep-th/0502139.

[9] K. M. Case, Phys. Rev., 80:5 (1950), 797-806.

[10] K. Meetz, Nuovo Cimento, 34 (1964), 690-708.

[11] Ф. М. Морс, Г. Фешбах, Методы теоретической физики, т. 2, ИЛ, М., 1960; М. Рид, Б. Саймон, Методы современной математической физики, т. 2: Гармонический анализ. Самосопряљсенность, Мир, М., 1978.

[12] D. M. Gitman, I. V. Tyutin, B. L. Voronov, Self-Adjoint Extensions in Quantum Mechanics: General Theory and Applications to Schrödinger and Dirac Equations with Singular Potentials, Progress in Mathematical Physics, 62, Birkhäuser, New York, 2012.

[13] F. Calogero, J. Math. Phys., 12:3 (1971), 419-436.

[14] М. Абрамовиц, И. Стиган (ред.), Справочник по специальным функциям с формулами, графиками и математическими таблицами, Наука, М., 1979.

[15] И. В. Тютин, частное сообщение.

[16] I. V. Tyutin, B. L. Voronov, Phys. Scr., 87:3 (2013), 038119, 14 pp., arXiv: 1211.6331; D. M. Gitman, I. V. Tyutin, B. L. Voronov, J. Phys. A: Math. Theor., 44:42 (2011), 425204, 17 pp., arXiv: 0907.1736.

[17] Э. Камке, Справочник по обыкновенным дифференциальным уравнениям, Наука, М., 1971.

[18] Л. Д. Ландау, Е. М. Лифшиц, Механика, Наука, М., 1988.

Поступила в редакцию 27.06.2014 